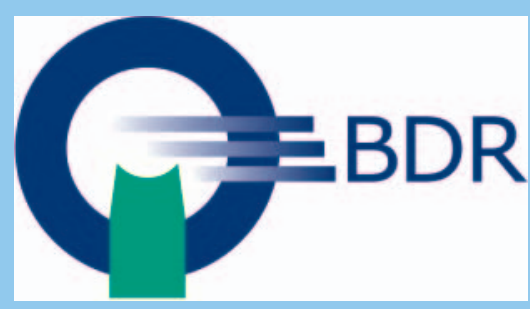

\title{
IROS 2019 - Von Kopf bis Fuß
}

Bereits zum vierten Mal öffnete der IROS seine Pforten im Januar im bcc Berlin am Alexanderplatz und feierte dort gleichzeitig sein 10-jähriges Bestehen in der heutigen Form. Das ursprünglich aus dem Olbert-Symposium hervorgegangene Treffen firmiert heute als Interventionell Radiologisches Olbert Symposium und wird als Dreiländertagung der Deutschen, Österreichischen und Schweizerischen Gesellschaften für Interventionelle Radiologie organisiert. Auch 2019 hatten sich die Organisatoren unter der Leitung der Kongresspräsidentin Prof. Maria Schoder (Wien) und des Co-Präsidenten Prof. Patrick Knüsel (Chur) viel vorgenommen. Insbesondere sollte die Fortentwicklung des Kongresses aus den vergangenen Jahren weiter vorangetrieben werden. Und so gab es auch heuer eine Vielzahl von neuen Formaten, Sitzungen und vor allem interaktiven Sitzungen zu erleben. Besonders zu erwähnen ist das sehr gut angenommene Format „Life-in-Box-Präsention“, bei der ein Videomitschnitt eines vorab aufgezeichneten interventionellen Eingriffs unter Moderation des Operateurs, der im Saal vor Ort ist, diskutiert wird. Im Gegensatz zum „echten“ LifeCase, hat diese Form der Präsentation den Vorteil, dass bei Fragen innerhalb des Falls problemlos angehalten und diskutiert werden kann und ggf. auch längere Prozeduren durch die Schnittmöglichkeiten innerhalb eines Sitzungsslots komplett und umfassend abgehandelt werden können.

Daneben wurde in der Eröffnungsveranstaltung auch gefeiert und zum Teil auf die Anfänge der Interventionellen Radiologie zurückgeblickt - ein wichtiger Programmpunkt, wenn man der Interventionellen Gesellschaft eine Kultur des Erinnerns und dem Kongress einen Rahmen für die Ehrung verdienter Mitglieder geben will. Vor allem jüngere Besucher fanden hier Anlass zur Kritik, da sie einen vergleichsweise langen

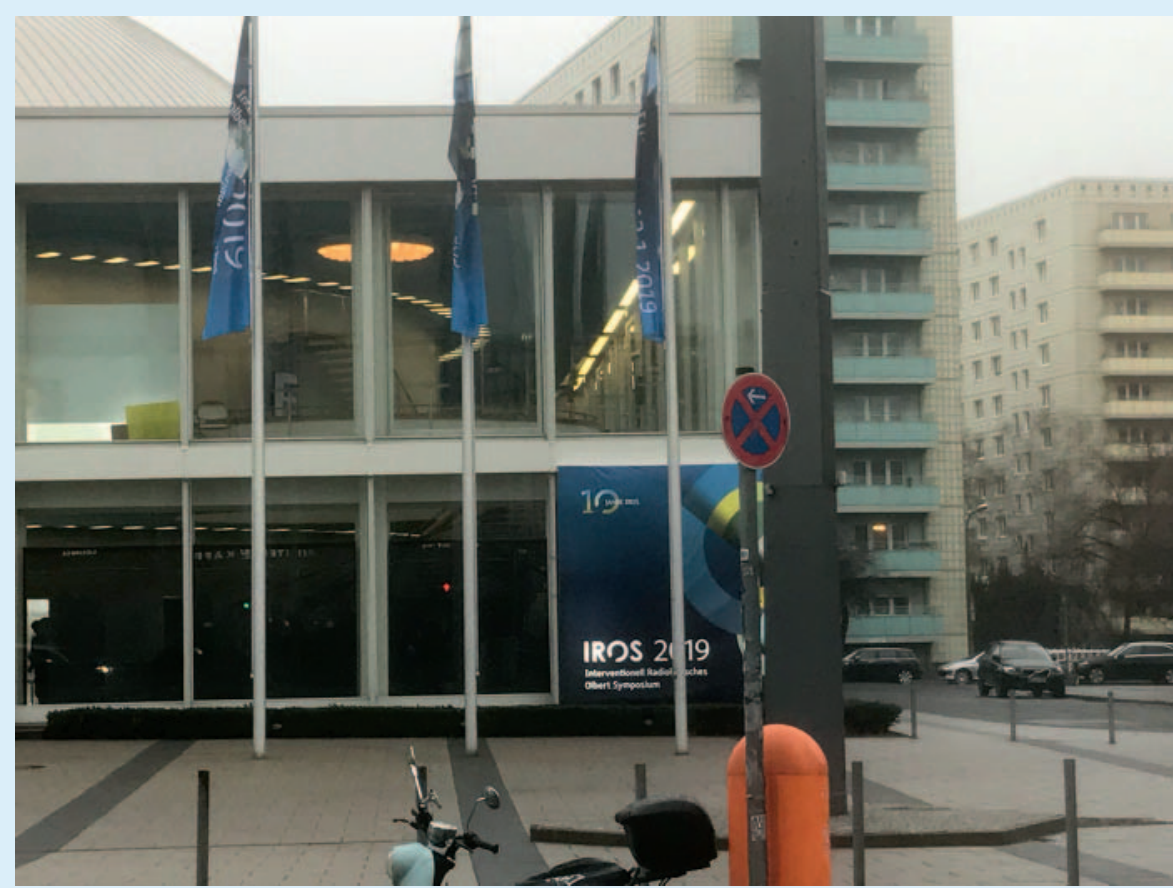

bcc - eine gut gewählte Kongress-Location. Copyright BDR

Zeitslot am ersten Tag vormittags lieber anders genutzt gesehen hätten. Das Format für den zukunftsfähigen Kongress wird sich entwickeln müssen.

Wie bereits in den Vorjahren ist ein Wandel bei den Teilnehmern zu verzeichnen. Neben den erfahrenen interventionell tätigen Radiologen, die den IROS gerne vor allem zum interkollegialen Austausch nutzen, kommen zunehmend junge Kollegen und MTRA. Hier ist es als großer Fortschritt anzusehen, dass an einer jährlich zunehmenden Zahl von interaktiven Kursen, einschließlich Simulatoren, Techniken erprobt und Fähigkeiten erweitert werden. Mit der zunehmenden Vielfalt interventioneller Verfahren können nicht alle Techniken ubiquitär in ausreichender Zahl auch gelehrt und ausgebildet werden. Ein strukturiertes Simulatortraining, wie unter der Initiative der DeGIR entwickelt und fortgeführt, ist hierfür beispielhaft und besonders begrüßenswert. Daneben erfreuen sich auch die Zertifizierungskurse der DeGIR einschließlich der Prüfungen ungebrochenen $\mathrm{Zu}$ spruchs. Dies gilt auch für die MTRA, die am zweiten Kongresstag ein eigenes Programm mit spezifischen interventionellen Themen durchgeführt haben.

Thematisch deckte der IROS 2019 tatsächlich alles „Von Kopf bis Fuß“ ab: an anatomischen Regionen ebenso wie im übertragenen Sinne in der Berücksichtigung aller interventionellen Verfahren. Besonders hervorzuheben ist dabei der auf Initiative der Veranstalter eingerichtete Ausstellungsbereich zum Strahlenschutz in der Interventionellen Radiologie - nachempfunden dem „Radiation Protection Pavillion“ bei CIRSE und ECR. Nicht nur wegen der europaweiten 
und in Deutschland in Form der novellierten Strahlenschutzverordnung geänderten gesetzlichen Grundlagen, sondern vor allem wegen der erheblichen Bedeutung für Untersucher und Patienten bei zunehmend aufwändigeren, damit zeit- und dosisintensiveren interventionellen Verfahren.

Im Bereich der vaskulären Interventionen spielen seit der flächendeckenden Einführung der Gefäßrekanalisation beim Schlaganfall neuroradiologische Themen eine zunehmend wichtigere Rolle und sind inzwischen auch in allen Formaten des Kongresses vertreten. Daneben wurde die Diskussion über weite Strecken durch die kürzlich erschienene Arbeit zu medikamentenbeschichteten Ballonen (DEB) und die dort publizierte erhöhte Todesrate nach Anwendung der Ballone diskutiert (Katsanos K. et al., JAHA Dez. 2018). Angesichts der in vielen Studien nachgewiesenen unbestreitbaren Vorteile des Verfahrens, waren sich alle Diskussionsteilnehmer einig, dass eine breitere Datenbasis erforderlich ist, um das Verfahren der DEB abschließend (be)werten zu können. Letztlich auch diesem Themenkreis zugehörig ist die seit einigen Jahren wieder verstärkt geführte Diskussion der „lesion-preparation“ zu Ungunsten des primären Stentings. Und auch die aktuellen Ergebnisse, die die Implantation von Stentgrafts im Bereich von Beckenstenosen als positiver erkannten als reine Metallstents. Neben der erhöhten Sicherheit für den Patienten dank ausgeschlossener Perforationsgefahr, hat diese Vorgehensweise den Vorteil des verbesserten Rekanalisationsdurchmessers bei verkalkten Stenosen aufgrund möglicher höherer Entfaltungsdrucke. Für die Unterschenkelrekanalisation wurde einmal mehr die Notwendigkeit der Herstellung einer kompletten Gefäßachse bis in die Fußperipherie durch mindestens ein Gefäß betont. Zur Erreichung dieses Ziels können ggf. auch alternative Punktionsmethoden von distal retrograd in unterschiedlichen Varianten beitragen.
Bei den Embolisationstechniken stand an etablierten Verfahren die SIRT erneut im Zentrum des Interesses. Angesichts unverändert sehr ernüchternder Literaturergebnisse sollte diese Therapiemethode derzeit, wenn irgend möglich unter Studienbedingungen und unter strenger standardisierter Indikationsstellung angewandt werden. Bei den derzeitigen Anwendern herrschte weitgehende Einigkeit, dass als Ursache der Ergebnisse auch die negative Patientenselektion aufgrund der fehlenden Verankerung der SIRT in den etablierten Leitlinien zu sehen ist. Zusammen mit der in Deutschland uneinheitlichen Kostenübernahme durch die Krankenkassen hat das Verfahren daher bisher nicht Eingang in klassische onkologische Therapieabläufe gefunden. Neue Impulse könnten sich durch eine Kombination mit neuen Formen der Immuntherapie ergeben. Daneben wurde die Embolisationstherapie benigner und maligner Prostatatumoren ausführlich diskutiert. Unabhängig von der technischen Machbarkeit ist die Rolle der Embolisationstherapie im Konzert urologischer und onkologischer Konzepte derzeit noch nicht ausreichend geklärt. Eine vollständig eigene Sitzung war Indikation, Technik und Outcome der Pfortaderembolisation im Rahmen der Leberchirurgie gewidmet. Unabhängig von lokalen Gegebenheiten ist der Stellenwert der interventionellen Pfortaderembolisation ggü. aufwändigen chirurgischen Verfahren auch 2019 nicht abschließend zu beantworten.

Dankenswerter Weise hatten die Veranstalter auch eine Sitzung der Kontrastmittelapplikation und ihrer Einschränkung bei reduzierter Nierenfunktion gewidmet. Einmal mehr wurde von nephrologischer Seite betont, dass bei anzustrebender möglichst geringer Kontrastmittelmenge dennoch eine indizierte Untersuchung nicht an der mangelnden Aussagekraft durch eine verwehrte Kontrastmittelgabe scheitern darf.
Wie in den vergangenen Jahren stellten die Formate „Ein Fall, der mich nicht schlafen ließ“ jeweils als Abschluss der ersten beiden Kongresstage und die zum Finale der Veranstaltung am Samstag-Mittag präsentierte „M\&M-Konferenz“ Publikumsmagnete dar. Vermutlich auch deshalb, weil neben schicksalshaften Verläufen in vielen der präsentierten Fallbeispiele aus dem langsam entgleitenden Verlauf entstehende Komplikationen geschildert wurden. Womit sich für alle Teilnehmer eine gute Gelegenheit ergibt, eigenes Handeln einzuordnen und ggf. neu zu bewerten.

Als Fazit bleibt, dass der IROS neben vielen vertrauten Funktionen wie persönlichem Erfahrungsaustausch, Präsentation neuester Daten oder Produkte in der Industrieausstellung und Möglichkeit des interventionellen Updates von Kopf bis Fuß vor allem auch seine Bedeutung für die Ausund Weiterbildung und den Zertifizierungsprozess unterstrichen hat. Er stellt damit ein deutschsprachiges und deutlich nationaleres Pendant mit eigenem Klientel zur inzwischen zum interventionellen Weltkongress mutierten CIRSE dar. Perspektivisch wird seine Bedeutung im radiologischen Kongresskalender daher, nicht nur wegen des Zeitversatzes von etwa sechs Monaten, weiter zunehmen.

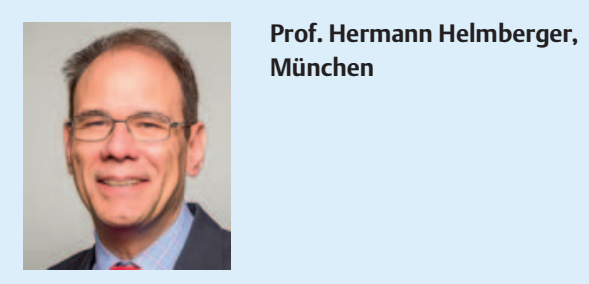

